\title{
Development and experimental validation of downlink multiuser MIMO-OFDM in gigabit wireless LAN systems
}

\author{
Koichi Ishihara*, Yusuke Asai, Riichi Kudo, Takeo Ichikawa, Yasushi Takatori and Masato Mizoguchi
}

\begin{abstract}
Multiuser multiple-input multiple-output (MU-MIMO) has been proposed as a means to improve spectrum efficiency for various future wireless communication systems. This paper reports indoor experimental results obtained for a newly developed and implemented downlink (DL) MU-MIMO orthogonal frequency division multiplexing (OFDM) transceiver for gigabit wireless local area network systems in the microwave band. In the transceiver, the channel state information (CSI) is estimated at each user and fed back to an access point (AP) on a real-time basis. At the $\mathrm{AP}$, the estimated $\mathrm{CSI}$ is used to calculate the transmit beamforming weight for $\mathrm{DL}$ MU-MIMO transmission. This paper also proposes a recursive inverse matrix computation scheme for computing the transmit weight in real time. Experiments with the developed transceiver demonstrate its feasibility in a number of indoor scenarios. The experimental results clarify that DL MU-MIMO-OFDM transmission can achieve a 972-Mbit/s transmission data rate with simple digital signal processing of single-antenna users in an indoor environment.
\end{abstract}

Keywords: Multiuser MIMO, OFDM, Downlink, Wireless LAN, CSI

\section{Introduction}

Wireless local area network (LAN) systems have recently seen explosive growth as the prevailing smart devices, e.g., smartphones and tablet computers, deploying wireless LAN chips to enable swift wireless access anywhere. In wireless LANs, broadband services such as video streaming and IP-TV services are becoming pivotal use cases. To provide such broadband services, ever higher data rate is required for wireless LANs. Spectrum efficiency improvement is becoming the most prioritized issue to meet the demand within the limited available frequency channels. Multiple-input multiple-output (MIMO) is one of the key technologies for this purpose; it improves the spectrum efficiency by enabling multiple spatial streams to be simultaneously transmitted from multiple transmit antennas. In the latest wireless access standards, i.e., IEEE 802.11n [1], single-user MIMO (SU-MIMO) with four spatial streams is specified to offer the maximum physical layer data rate of $600 \mathrm{Mbit} / \mathrm{s}$ in $40-\mathrm{MHz}$ bandwidth.

* Correspondence: ishihara.koichi@lab.ntt.co.jp

NTT Network Innovation Laboratories, NTT Corporation, Yokosuka, Japan
It also adopts orthogonal frequency division multiplexing (OFDM) to mitigate the effect of multipath fading, resulting in reduced calculation complexity by enabling the MIMO signal processing to be carried out on a persubcarrier basis. For future wireless communication systems, the multiuser MIMO (MU-MIMO) technique is an attractive candidate to achieve higher spectrum efficiency, especially when the number of antenna branches on the user side is less than that on the access point (AP) [2]. Although SU-MIMO can also improve spectrum efficiency by increasing the number of transmit and receive antennas, the number of antenna branches on the user side is limited in terms of portable device size. In contrast, MU-MIMO makes it possible to multiplex users in the spatial domain so that higher frequency efficiency can be expected even when using simple users. There have been many studies pertaining to MU-MIMO systems, e.g., [3-5]. The next generation wireless LAN system targeting more than $1 \mathrm{Gbit} / \mathrm{s}$ is studied in IEEE 802.11ac, and downlink (DL) MU-MIMO-OFDM has been extensively investigated as a means to improve the spectrum efficiency [6,7]. In computing the transmit 
beamforming weight at the AP transmitter, higher accuracy is required in DL MU-MIMO transmission than in SU-MIMO transmission. This higher accuracy is required to suppress the inter-stream interference between users since users cannot detect other users' data, especially with the type of simple decoder used in wireless LANs. Furthermore, in IEEE 802.11ac, the transmission bandwidth mandatorily becomes $80 \mathrm{MHz}$, and the number of transmission antennas is defined as being up to eight, resulting in increased calculation complexity. Therefore, it is important to use implemented hardware to confirm the feasibility of DL MU-MIMO under such actual conditions for wireless LANs. In previous work in this area, an implemented transceiver has been used to experimentally validate SU-MIMO techniques such as eigenbeam space division multiplexing [8-10]. In [11], an implementation scheme of a nonlinear precoding based on TomlinsonHarashima Procoding for MU-MISO-OFDM has been proposed. This scheme improves the transmission performance by iterative operation.

In this paper, we present indoor experimental results obtained with a newly developed and implemented realtime DL MU-MIMO-OFDM transceiver using fieldprogrammable gate arrays (FPGAs) for the next generation wireless LAN systems. The transmission system consists of an AP with eight antenna elements and six users each having a single antenna element; the transmission bandwidth is $80 \mathrm{MHz}$. In DL MU-MIMO transmission, channel state information (CSI) is required to compute the transmit beamforming weight at the AP transmitter. In the transceiver, closed-loop feedback is used in accordance with the IEEE 802.11ac Draft. A sounding frame is used to estimate CSI at each user, and each user feeds it back to an AP on a real-time basis. The CSI is used to calculate the transmit beamforming weight at the AP, and the DL MU-MIMO transmission is then carried out via real-time digital signal processing. For computing the transmit beamforming weight in real time, we propose a recursive inverse matrix computation (RIMC) scheme based on a recursive least square (RLS) algorithm [12], in which the inverse of the auto-correlation matrix of the CSI for beamforming weight computation is calculated over successive time intervals. In the proposed scheme, the inverse matrix can be calculated in the arrival order of CSI feedback frames from each user in a time-domain multiple access (TDMA) manner, resulting in reduced overhead for the transmit beamforming weight computation. Moreover, since the transmit beamforming weight obtained by the proposed RIMC scheme is based on zero-forcing (ZF) beamforming, it can ideally provide orthogonal transmission between each stream, and users can decode their own data with a simple decoder in a system such as a single-input singleoutput (SISO) system. The ZF weight proposed in our paper is a linear precoding scheme that does not require iterative operation, whereas the conventional scheme [11] requires iterative operation to improve the transmission performance. Furthermore, while there is no limitation on the number of transmit antennas to compute the ZF weight in the proposed scheme, the conventional one can compute it only in two-by-two MIMO. Since the maximum number of transmit antennas will be defined as eight in 11ac, the proposed scheme is valid for the next generation wireless LAN. In our previous work [13], we showed experimental results obtained with our developed and implemented transceiver in an experimental room. In this paper, we show not only these results but also two additional experimental results obtained with our developed and implemented transceiver in a large office and a small apartment. The propagation environments are different among these three places. It is important to evaluate the transmission performance of DL MU-MIMO in different environments since the transmit beamforming is affected by the propagation channel. Performance evaluation for time-varying channels is also described as new results. Since the estimated CSI becomes outdated for DL MU-MIMO transmission, the imperfect CSI causes inter-user interference. However, there have been no reports on real-time evaluations of DL MU-MIMO-OFDM transmission performance in time-varying channels. In this paper, we also evaluate the transmission rate obtained with our transceiver in such a channel and verify the feasibility of DL MU-MIMOOFDM for gigabit-per-second transmission in wireless LAN systems. The paper also mentions information relevant to implementation such as types of FPGA, maximum frequency, and intervals of frame sequence, which show that DL MU-MIMO-OFDM is a promising technology to enable gigabit-per-second transmission for simple terminals with feasible hardware implementation at the AP and users sides.

The remainder of this paper is organized as follows. We explain the transceiver configuration in Section 2. In Section 3, we describe the experimental results we obtained with the transceiver in indoor scenarios. Finally, our conclusions are presented in Section 4.

The following notations will be used throughout this paper: Column vectors (matrices) are denoted by boldface lower (upper) case letters, superscripts ' $\mathrm{T}$ ' and ' $\mathrm{H}$ ' respectively stand for transpose and conjugate transpose, and $\mathbf{I}_{n}$ denotes an $n \times n$ identity matrix.

\section{Configuration of DL MU-MIMO-OFDM transceiver} 2.1. Transceiver overview

Figures 1 and 2, respectively, show the DL MU-MIMOOFDM transmitter and receiver configurations. The major DL MUMIMO-OFDM parameters are listed in Table 1. The carrier frequency is $4.85 \mathrm{GHz}$, and the maximum number of users for DL MU-MIMO-OFDM transmission 


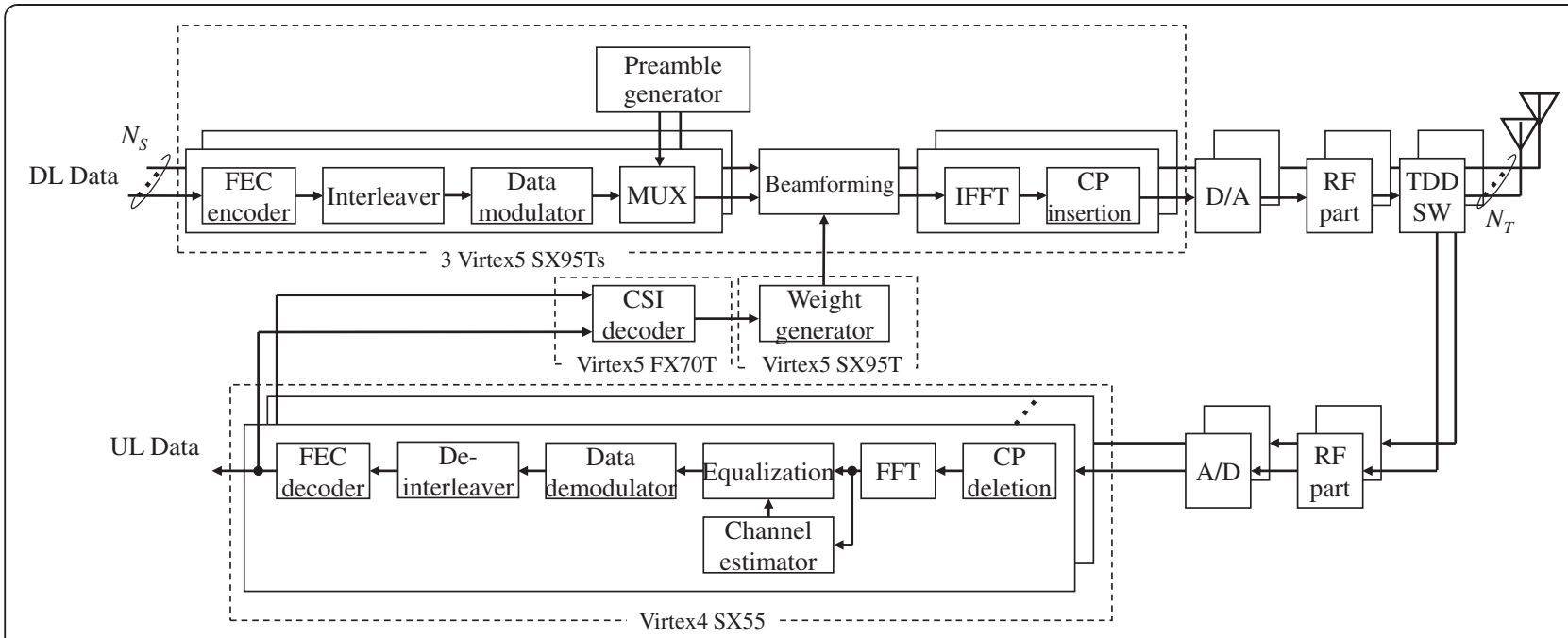

Figure 1 DL MU-MIMO transceiver structure at the AP side.

is six. In this transceiver, $N_{\mathrm{s}}$-independent streams are transmitted simultaneously, where $N_{\mathrm{s}}$ is the number of users. The number of subcarriers is 228, which includes 12 pilot subcarriers. At the AP transmitter, the number of antennas $N_{\mathrm{T}}$ is eight, and eight-antenna OFDM signals with $80-\mathrm{MHz}$ transmit bandwidth are generated. At each user's receiver, the signal from the AP is received at an antenna. Channel coding is convolutional coding with $R=1 / 2$ coding rate. Digital signal processing at both the AP and user sides is carried out in FPGAs. Table 2 shows a list of the utilized Xilinx Virtex FPGAs (Xilinx, Inc., San Jose, CA, USA) [14] for our implemented DL MU-MIMO. At the AP in our implemented transceiver, six FPGAs are used for signal processing in DL MU-MIMO transmission: a Virtex5 SX95T for weight computation for DL MUMIMO, three Virtex5 SX95Ts for transmitter signal processing, a Virtex5 FX70T for controlling the previous four Virtex5 SX95Ts and for obtaining CSI, and a Virtex4 SX55 for receiver signal processing. At the users' side in our transceiver, eight FPGAs are used in all: two Virtex5 SX95Ts are used for transmitter signal processing and each processes the transmission signals of the three users, with the processing being carried out independently for each user. Each Virtex4 SX55 is used to process the receiver signals of each user. The maximum clock frequency in our transceiver is $80 \mathrm{MHz}$.

\subsection{Frame sequence structure}

Figure 3 shows the frame sequence structure in the transceiver. The frame sequence consists of a sounding frame, CSI feedback (CSI-FB) frames, and a data frame. For DL MU-MIMO-OFDM transmission, the CSI from the AP to each user is required to perform the transmit beamforming at the AP's transmitter. In the transceiver, the CSI is estimated using the sounding frame at each user, and it is fed back to the AP.

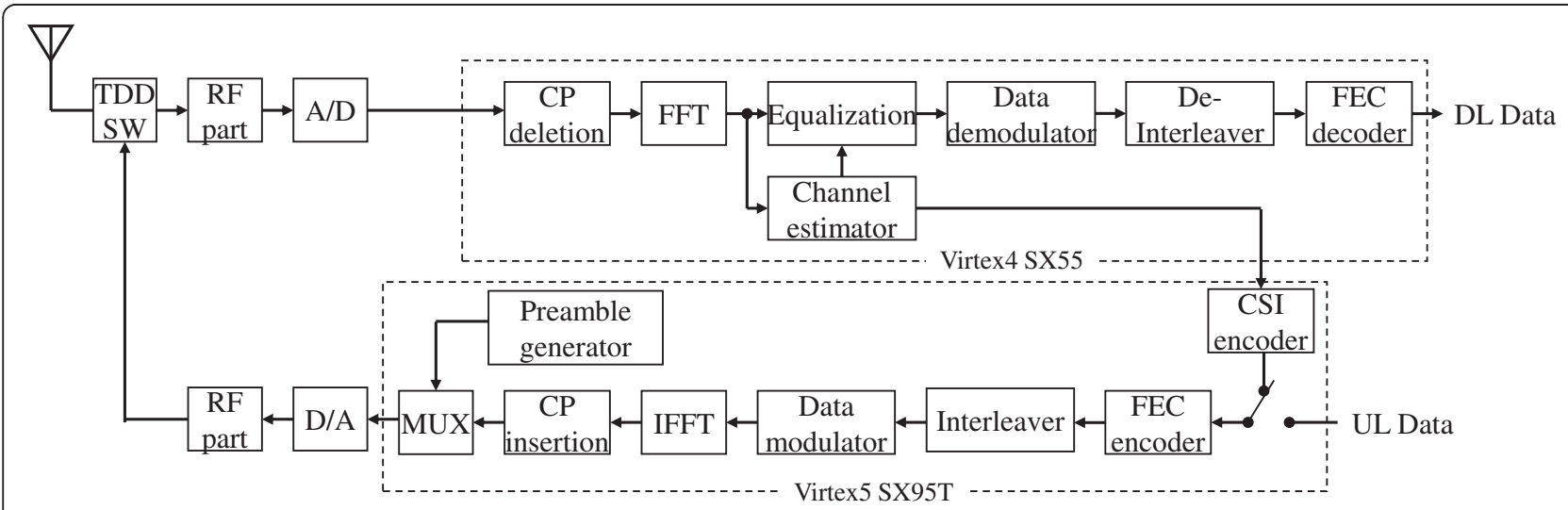

Figure 2 DL MU-MIMO transceiver structure at the user side. 


\begin{tabular}{lc}
$\begin{array}{l}\text { Table } \mathbf{1} \text { Major parameters in } \\
\text { transceiver }\end{array}$ & Unit \\
\hline Parameter & $80 \mathrm{MHz}$ \\
\hline Bandwidth & $4.85 \mathrm{GHz}$ \\
Carrier frequency & $8(\mathrm{AP}) / 1$ (user) \\
Number of antennas & 6 \\
Maximum number of users & $30 \mathrm{dBm}$ \\
Maximum total transmit power & 228 \\
Number of subcarriers & (with 12 pilot subcarriers) \\
OFDM symbol duration & $4.0 \mu \mathrm{s}$ \\
Data modulation & $(3.2 \mu \mathrm{s}+\mathrm{CP} 0.8 \mu \mathrm{s})$ \\
Channel coding/decoding & QPSK, $16 \mathrm{QAM}, 64 \mathrm{QAM}$ \\
& Convolutional coding $(R=1 / 2)$ Niterbi \\
CP Cyclic prefix. & decoding
\end{tabular}

At the AP, a sounding frame is first transmitted to each user. Figure 4 shows the sounding frame structure. The frame consists of a short preamble and a long preamble. Both preambles are constructed from the $40-\mathrm{MHz}$ design defined in IEEE 802.11n [15] by duplicating and frequency shifting. The long preamble uses an $8 \times 8$ Hadamard code matrix [16], and eight OFDM symbols are transmitted to estimate the $8 \times 1$ CSI vector from eight transmit antennas at the AP to an antenna at each user.

At each user, the CSI vectors are estimated using the received sounding frame at each subcarrier. In-phase and quadrature-phase quantization are then performed on each CSI estimate. The quantized CSI is encoded using convolutional coding. After interleaving and data modulation mapping, 256-point inverse fast Fourier transform (IFFT) is used to convert the data symbol sequence into an OFDM signal with 228 subcarriers. Then, the OFDMmodulated CSI is transmitted with short and long preambles to the AP as a CSI-FB frame. Here, in uplink transmission, a CSI-FB frame is transmitted from each user in a TDMA manner.

After receiving the CSI-FB frame, the transmit beamforming weight based on ZF beamforming is computed at each subcarrier at the AP for DL MU-MIMO-OFDM transmission. The weight computation scheme will be described in the next section. After computing the beamforming weight, the transmitted DL MU-MIMOOFDM signal is generated. First, a data sequence is encoded using convolutional coding with the coding rate $R=1 / 2$ and interleaving is carried out for each user. After data modulation and pilot (short and long preambles) insertion, the transmit beamforming is performed in both the data and pilot sequence using the computed weight at each subcarrier. Figure 5 shows the data subframe structure. As the figure shows, the subframe's long preamble consists of $2 N_{\mathrm{s}}$ OFDM symbols, and the transmit beamforming for each user is performed in each OFDM symbol to obtain an interference-free CSI estimate at each user. The 256-point IFFT is then applied to produce the time-domain OFDM symbol for each transmitted stream. After cyclic prefix insertion, the DL MUMIMO-OFDM signal sequences are transmitted from eight antennas at the AP.

At the user, after timing synchronization and frequency offset compensation, channel estimation is carried out using the long preamble at each subcarrier. The channel estimates are used to equalize and demodulate all the data symbols within the subframe. The conventional phase tracking scheme proposed in [17] is also applied to all the data symbols by using the pilot subcarriers. Here, in the transceiver, simple decoding can be carried out as in SISO transmission at each user. This is because ZF-based transmit beamforming can provide orthogonal transmission between each stream ideally, and each user can simply decode the received signal even with a single antenna. After data demodulation, the transmission binary data is obtained through Viterbi decoding, where the number of bits for soft decision at the Viterbi decoder is three due to hardware implementation limitations.

Table 3 shows the duration of each frame and interval. In terms of signal processing, the differences between DL MU-MIMO transmission in 11ac and the conventional transmission in wireless LANs such as that defined in $11 \mathrm{n}$ are CSI feedback and DL MU-MIMO signal generation including weight computation. Therefore, we discuss each of these separately. First, we discuss the CSI feedback. Both CSI estimation and CSI-FB frame generation are carried

Table 2 The list of the utilized Xilinx FPGAs for our implemented DL MU-MIMO transceiver

\begin{tabular}{ccccc}
\hline & Model & Unit & Application & Occupied slices (\%) \\
\hline AP & Virtex5 SX95T & 1 & Weight computation & 90 \\
& Virtex5 SX95T & 3 & Transmitter signal processing & 95 \\
& Virtex5 FX70T & 1 & Controlling four Virtex5 SX95T, CSI acquisition & 67 \\
Six users & Virtex4 SX55 & 1 & Receiver signal processing & 83 \\
& Virtex5 SX95T & 2 & Transmitter signal processing & 56 \\
& Virtex4 SX55 & 6 & Receiver signal processing & 90 \\
\hline
\end{tabular}




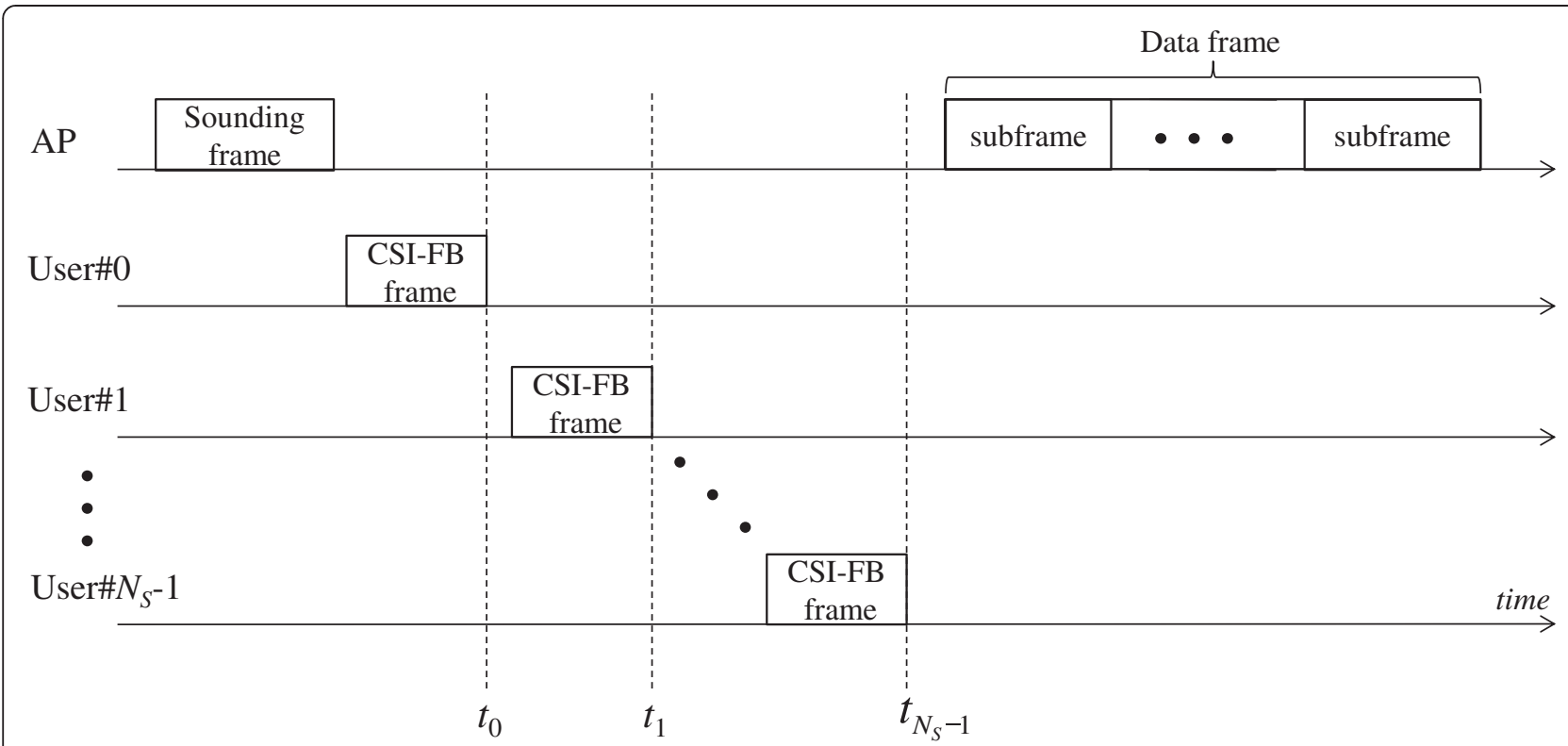

Figure 3 Frame sequence structure in the transceiver. The frame sequence consists of a sounding frame, CSI feedback frames, and a data frame.

out during the interval (b) in Figure 3, which takes $70 \mu$ s. In our transceiver, the CSI-FB frames are transmitted after all the CSI-FB frames for six users are generated for uplink TDMA transmission. Therefore, the actual interval for each user is $70 / 6=11.7 \mu$ s. Here, the interval should be less than a short inter-frame space (SIFS) of $16 \mu \mathrm{s}$, which is the duration between two successive frames within a frame sequence. Thus, the CSI-FB frames can be transmitted within the SIFS duration. Second, we discuss the DL MU-MIMO signal generation including the weight computation. This is started at the end of the first CSIFB frame $t_{0}$ and finished by the beginning of the data frame. The total signal processing delay for six users is $368 \mu$ s. In 11ac, there is no stipulation about this since the CSI-FB frames and DL MU-MIMO data frames can be transmitted independently. However, as the delay becomes longer, transmission performance is degraded since the orthogonality between users is deteriorated due to time-varying channels. We will discuss the effect of interval (d) in the next section.

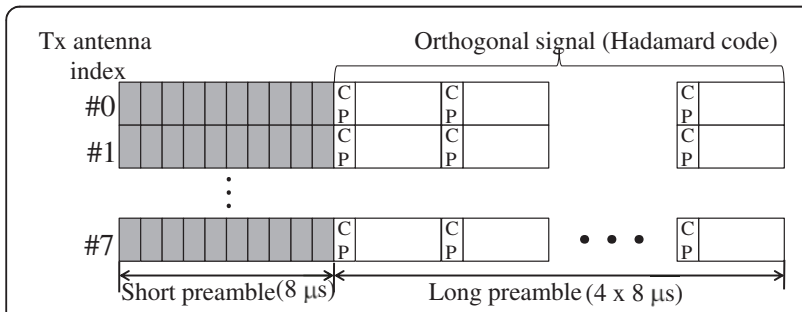

Figure 4 Sounding frame structure. The frame consists of a short preamble and a long preamble.

\subsection{RIMC scheme for DL MU-MIMO-OFDM}

In the transceiver, the beamforming weight is used for transmit beamforming. Generally, the weight is given by [18]

$$
\mathbf{W}(k)=\mathbf{H}(k)^{H}\left(\mathbf{H}(k) \mathbf{H}(k)^{H}+\delta \mathbf{I}_{N_{S}}\right)^{-1},
$$

where

$$
\mathbf{H}(k)=\left[\mathbf{h}_{0}(k)^{T}, \ldots, \mathbf{h}_{N_{S}-1}(k)^{T}\right]^{T} .
$$

Here, $\delta$ is a positive constant used to suppress the noise enhancement. The $\mathbf{h}_{m}(k)$ term is the $1 \times N_{T}$ CSI vector between the antenna at the $m$-th user and the multiple transmit antennas at the AP at the $k$-th subcarrier and is given by

$$
\mathbf{h}_{m}(k)=\left[H_{m, 0}(k), H_{m, 1}(k), \ldots, H_{m, N_{T}-1}(k)\right] .
$$

Here, $H_{m, n}(k)$ is the CSI estimate between the $n$-th antenna at the AP and the antenna at the $m$-th user at the

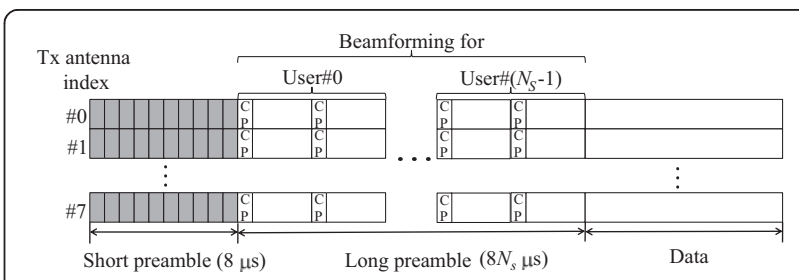

Figure 5 Data subframe structure. The subframe's long preamble consists of $2 N_{\mathrm{s}}$ OFDM symbols, and the transmit beamforming for each user is performed in each OFDM symbol to obtain an interference-free CSI estimate at each user. 
Table 3 Frame lengths and intervals in our implemented DL MU-MIMO transceiver

\begin{tabular}{cc}
\hline Position & Time $(\boldsymbol{\mu s})$ \\
\hline (a) & 44 \\
(b) & 70 \\
(c) & 60 \\
(d) & 318 \\
(e) & $8+8 N_{s}+4 n(n=1,2, \ldots)$ \\
(f) & $440+68 N_{s}+4 n(n=1,2, \ldots)$ \\
\hline
\end{tabular}

$k$-th subcarrier. As shown in Equation (1), to obtain the beamforming weight, it is necessary to compute the inverse of the auto-correlation matrix of the CSI. However, this increases the overhead for the weight computation. In this paper, an RIMC scheme is proposed for the DL MUMIMO-OFDM transceiver to reduce overhead in computing the beamforming weight [12]. If $\left[\mathbf{H}(k) \mathbf{H}(k)^{H}+\delta \mathbf{I}_{N_{S}}\right]$ is nonsingular, Equation (1) can be rewritten as

$$
\mathbf{W}(k)=\left(\mathbf{H}(k)^{H} \mathbf{H}(k)+\delta \mathbf{I}_{N_{T}}\right)^{-1} \mathbf{H}(k)^{H} .
$$

Here, $\mathbf{R}(k)$ and $\mathbf{R}_{m}(k)$ are defined as

$$
\begin{aligned}
\mathbf{R}(k) & =\left(\mathbf{H}(k)^{H} \mathbf{H}(k)+\delta \mathbf{I}_{N_{T}}\right)^{T} \\
& =\sum_{l=0}^{N_{S}-1} \mathbf{h}_{l}(k)^{H} \mathbf{h}_{l}(k)+\delta \mathbf{I}_{N_{T}} \\
\mathbf{R}_{m}(k) & =\sum_{l=0}^{m} \mathbf{h}_{l}(k)^{H} \mathbf{h}_{l}(k)+\delta \mathbf{I}_{N_{T}}
\end{aligned}
$$

where $\mathbf{R}_{-1}(k)=\delta \mathbf{I}_{N_{T}}$ and $\mathbf{R}_{N_{S}-1}(k)=\mathbf{R}(k)$. The $\mathbf{R}_{m}(k)$ term can be expressed as the following recursive form:

$$
\mathbf{R}_{m}(k)=\mathbf{R}_{m-1}(k)+\mathbf{h}_{m}(k)^{H} \mathbf{h}_{m}(k)
$$

From a formulation of the matrix inversion in [19], we can obtain the inverse matrix of $\mathbf{R}_{m}(k)$ as

$$
\mathbf{R}_{m}(k)^{-1}=\mathbf{R}_{m-1}(k)^{-1}-\frac{\mathbf{R}_{m-1}(k)^{-1} \mathbf{h}_{m}(k)^{H} \mathbf{h}_{m}(k) \mathbf{R}_{m-1}(k)^{-1}}{1+\mathbf{h}_{m}(k) \mathbf{R}_{m-1}(k)^{-1} \mathbf{h}_{m}(k)^{H}} .
$$

Here, the vector $\mathbf{p}_{m}(k)$ and the scalar $q_{m}(k)$ are defined as

$$
\begin{aligned}
& \mathbf{p}_{m}(k)=\mathbf{R}_{m-1}(k)^{-1} \mathbf{h}_{m}(k)^{H} \\
& q_{m}(k)=1+\mathbf{h}_{m}(k) \mathbf{p}_{m}(k)
\end{aligned}
$$

From Equations (9) and (10), Equation (8) is rewritten as

$$
\mathbf{R}_{m}(k)^{-1}=\mathbf{R}_{m-1}(k)^{-1}-\frac{\mathbf{p}_{m}(k) \mathbf{p}_{m}(k)^{H}}{q_{m}(k)} .
$$

Since the CSI-FB frames transmitted from the users are received at the AP in a TDMA manner, calculating the inverse of the auto-correlation matrix progresses in the CSI arrival order. If the time that the CSI of the $m$-th user obtained is $t_{m}$, the calculations of the vector $\mathbf{p}_{m}(k)$ and the scalar $q_{m}(k)$ in Equations (9) and (10) are started at $t_{m}$, and $\mathbf{R}_{m}(k)^{-1}$ is updated. According to the above calculation, $\mathbf{R}_{m}(k)^{-1}$ is carried out iteratively until $m=N_{\mathrm{s}}-1$. Finally, using the obtained CSI matrix and the inverse of the auto-correlation matrix $\mathbf{R}(k)^{-1}=\mathbf{R}_{N_{S^{-1}}}(k)^{-1}$, the transmit beamforming weight $\mathbf{W}(k)$ is obtained as

$$
\mathbf{W}(k)=\left(\mathbf{R}(k)^{-1}\right)^{T}\left[\begin{array}{c}
\mathbf{h}_{0}(k) \\
\vdots \\
\mathbf{h}_{N_{S}-1}(k)
\end{array}\right]^{H}
$$

In the proposed RIMC scheme, an inverse matrix computation is carried out recursively by using an RLS algorithm, and the computation circuits of $\left\{\mathbf{R}_{m}(k)^{-1}, m=\right.$ $\left.0, \ldots, N_{\mathrm{s}}-1\right\}$ can be communalized. As a result, the circuit size can be reduced. Furthermore, since each CSIFB frame is transmitted from each user in order in 11ac, the proposed scheme can successively calculate the inverse matrix according to the CSI arrival order from each user. Thus, the AP can start the ZF weight computation at time $t_{1}$ in Figure 3 and successively calculate the weight by using every CSI-FB frame. This algorithm can reduce the overhead in calculating the transmit beamforming weight and can hasten the start of the DL MU-MIMO transmission. This leads to small performance degradation due to channel time varying.

\section{Experimental results}

Figure 6 shows the indoor measurement environment in an experimental room. The AP antenna arrays had the element spacing of $0.06 \mathrm{~m}(\approx 1.0 \lambda$, where $\lambda$ is the wavelength), and the measured value of the spatial correlation between the adjacent transmit antennas was 0.16. The antenna heights for the AP and for the users were, respectively, 1.5 and $1.0 \mathrm{~m}$. The total maximum transmit power was $30 \mathrm{dBm}$ and was allocated to each stream equally. We used linear arrays with eight antennas at the AP and a single antenna at each user. The same assortment of modulation schemes, i.e., QPSK, 16QAM, and 64QAM, was applied to all streams (or users). The coding rate was set at $R=1 / 2$. The data frame length is $5.12 \mathrm{~ms}$, and the frames consist of 32 subframes. In the following experiment, the number of 


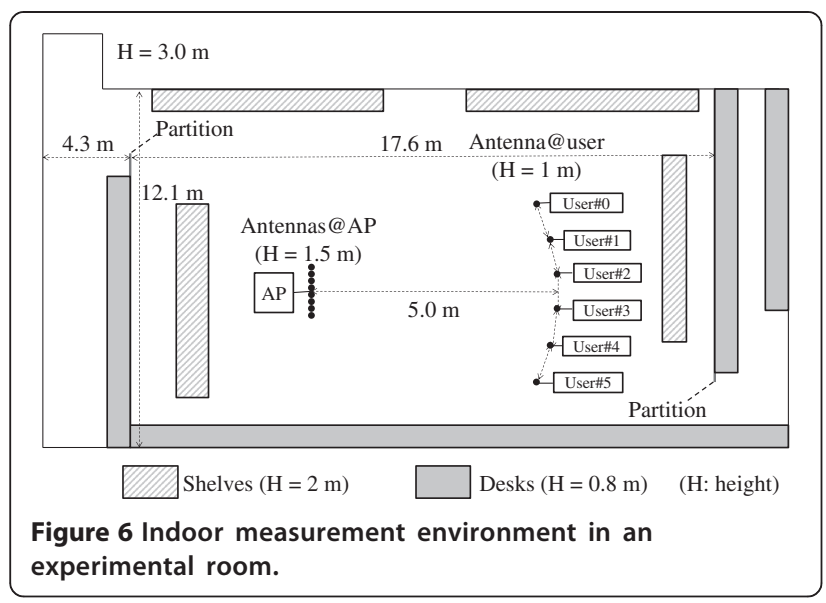

feedback information bits for CSI $\operatorname{Re}\left[H_{m, n}(k)\right]$ or Im $\left[H_{m, n}(k)\right]$ is 12 ; the CSI-FB uplink frames were transmitted through wired transmission in a TDMA manner, and there is no propagation error in the CSI feedback. Figure 7 shows an example of estimated channel impulse response in the experimental room. We evaluated the channel impulse response by applying inverse discrete Fourier transform (IDFT) to the estimated CSI at each subcarrier in $20-\mathrm{MHz}$ bandwidth since the preamble is constructed from the $40-\mathrm{MHz}$ design defined in $11 \mathrm{n}$ to $80 \mathrm{MHz}$, and there are null subcarriers between subcarrier groups of $20 \mathrm{MHz}$. Note that the right half of the IDFT output was omitted because it does not show the real channel impulse response. As shown in Figure 7, the measured value of the delay spread in the experimental room is 104.0 ns. Figure 8 plots the average transmission rate as a function of the average total transmit power of DL MU-MIMO-OFDM transmission with the three modulation schemes. The distance

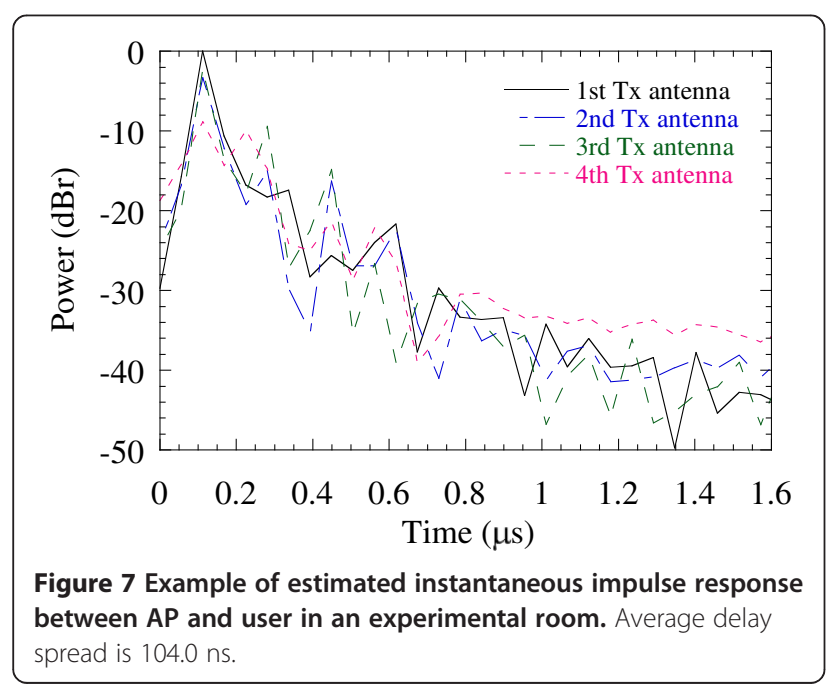

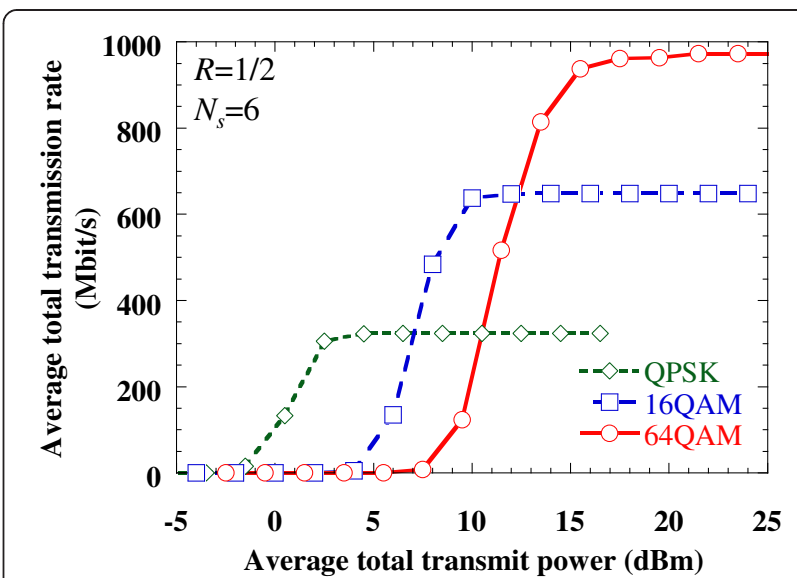

Figure $\mathbf{8}$ Average transmission rate. The rate is a function of the total transmit power of DL MU-MIMO transmission with three data modulation schemes.

between adjacent users is $2.0 \mathrm{~m}$. Here, the transmission rate of DL MU-MIMO-OFDM, $r$, is defined as

$$
r=\sum_{n=0}^{N_{s}-1} \frac{N_{\mathrm{sc}} R \log _{2} M}{T_{s}}\left(1-P_{n}\right)
$$

where $N_{\mathrm{sc}}$ is the number of data subcarriers, $M$ is the number of constellation points, $T_{\mathrm{s}}$ is OFDM symbol duration, and $P_{n}$ is packet error rate (PER) at the $n$-th user. As the figure shows, the transmission rates of 324, 648, and 972 $\mathrm{Mbit} / \mathrm{s}$ are achieved at the average transmit power of approximately 3,10, and $16 \mathrm{dBm}$ with the QPSK, 16QAM, and 64QAM modulation schemes, respectively. These results show that DL MU-MIMO-OFDM transmission with high-level modulation can be applied with a simple decoder at each user in an indoor environment and that a transmission rate over $972 \mathrm{Mbit} / \mathrm{s}$ can be achieved.

Next, Figure 9 shows the indoor measurement environment in a large office. There were many desks and partitions around the AP and the users, and each user was surrounded by partitions. The antenna heights for the AP and for the users were, respectively, 1.8 and 0.8 $\mathrm{m}$, and the distance between adjacent users was $2.5 \mathrm{~m}$. The AP antenna arrays had the element spacing of 0.03 $\mathrm{m}(\approx 0.5 \lambda)$, and the measured value of the spatial correlation between the adjacent transmit antennas was 0.47 . Each user antenna was set up on a desk. The total maximum transmit power was $24 \mathrm{dBm}$, and the modulation format and coding rate were set to 64QAM and $R=1 / 2$, respectively. In this experiment, we measured the transmission performance in two cases. In case 1 , people were working with a $\mathrm{PC}$ at a desk. In case 2 , a person was walking in front of the AP under the case 1 condition. Figure 10 shows an example of estimated instantaneous impulse response in the office. As the figure shows, 


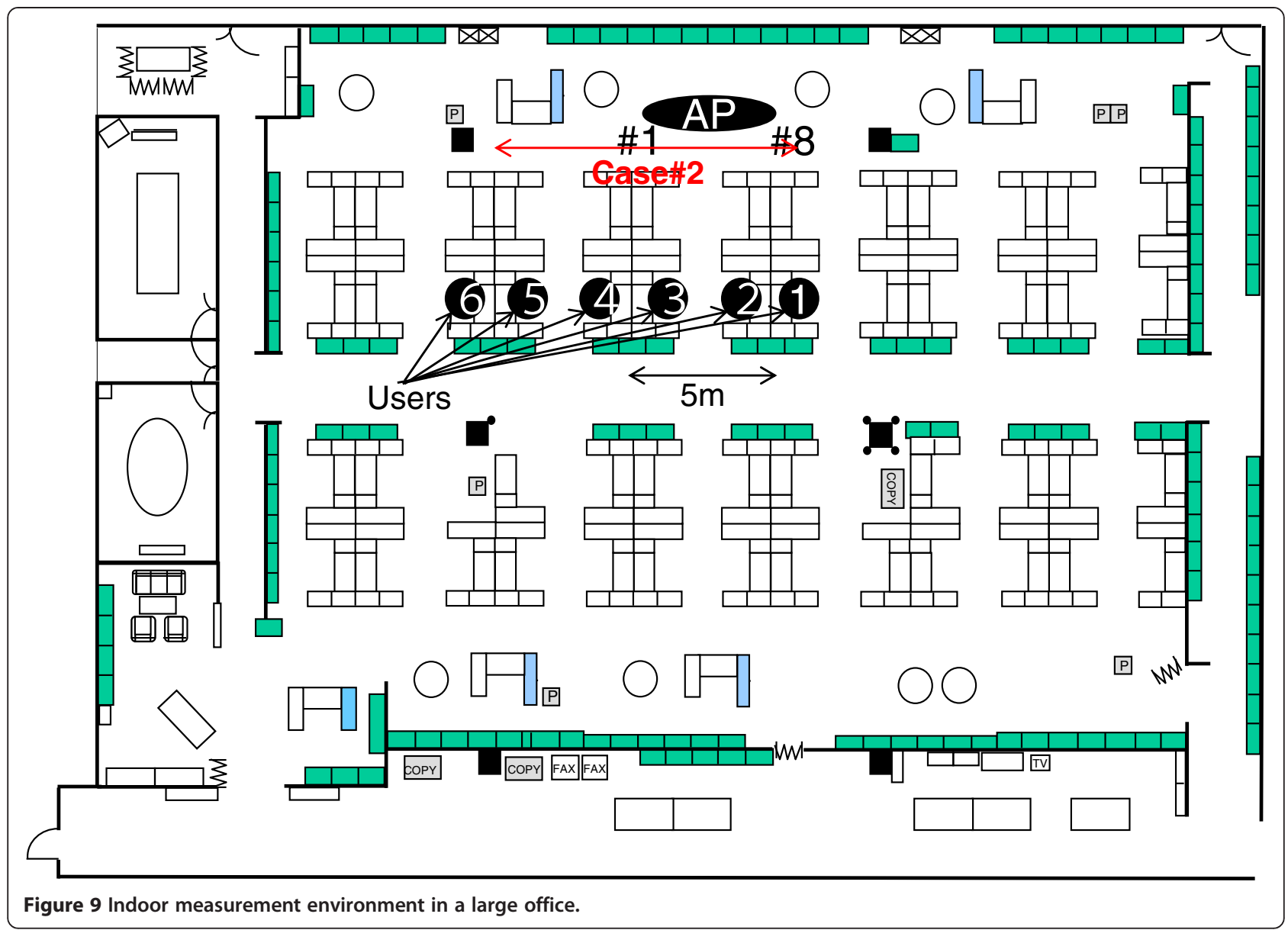

the measured value of the delay spread in the office is $99.8 \mathrm{~ns}$, which is almost the same as the $104.0 \mathrm{~ns}$ for the experimental room. The difference between them is the time-varying characteristic of the channel. The channel in the experimental room is quasi-static, whereas

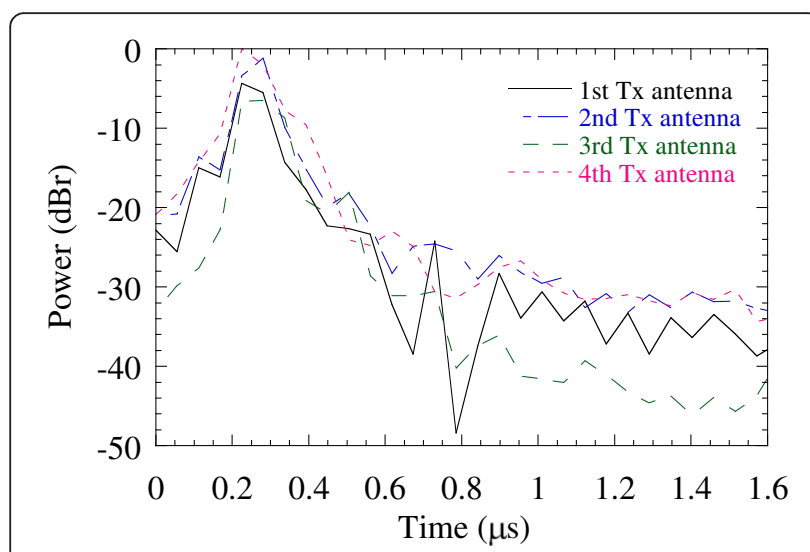

Figure 10 Example of estimated instantaneous impulse response between AP and user in a large office. Average delay spread is 99.8 ns. the channel in the office environment is time-varying. Figure 11 plots the average transmission rate as a function of the data frame length. As the data frame becomes longer, transmission performance is degraded since the orthogonality between users is deteriorated due to channel time varying. In particular, the transmission rate in case 2 is lower than that in case 1 because the propagation

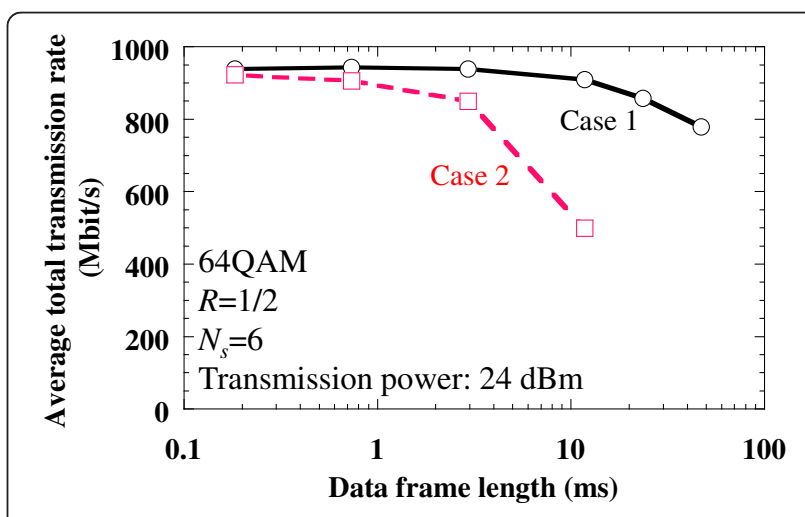

Figure 11 Effect of channel time varying. The average transmission rate is a function of the data frame length. 


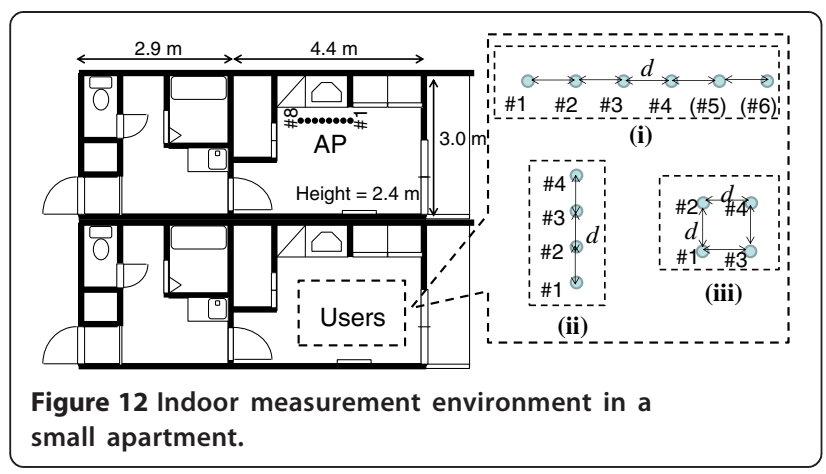

channel in case 2 varies faster than that in case 1 . However, no performance degradation can be seen until after 11.8 and $2.9 \mathrm{~ms}$ of the data frame length. These amount to 13.2 and $4.3 \mathrm{~ms}$ of the interval (f) in Figure 3 in cases 1 and 2, respectively. This means DL MU-MIMO-OFDM transmission is effective as long as CSI is fed back within these time lengths. Furthermore, the interval (d) of $318 \mu$ s is much less than the aforementioned data frame lengths, and the overhead is small. This means the interval is reasonable for DL MU-MIMO transmission.

Finally, we measured the transmission rate with the transceiver in a small apartment environment. Figure 12 shows the indoor measurement environment and the user locations in it. The antenna heights for the AP and for the users were 1.5 and $1 \mathrm{~m}$, respectively. The AP antenna arrays had the element spacing of $0.03 \mathrm{~m}(\approx 0.5 \lambda)$, and the measured value of the spatial correlation between the adjacent transmit antennas was 0.54 . The total maximum transmit power was $24 \mathrm{dBm}$, and the modulation format and coding rate were set to 64QAM and $R=1 / 2$, respectively. The data frame length is $5.12 \mathrm{~ms}$, and frames consist of 32 subframes. As shown in the figure, the users were located so as to maximize the angle between the AP

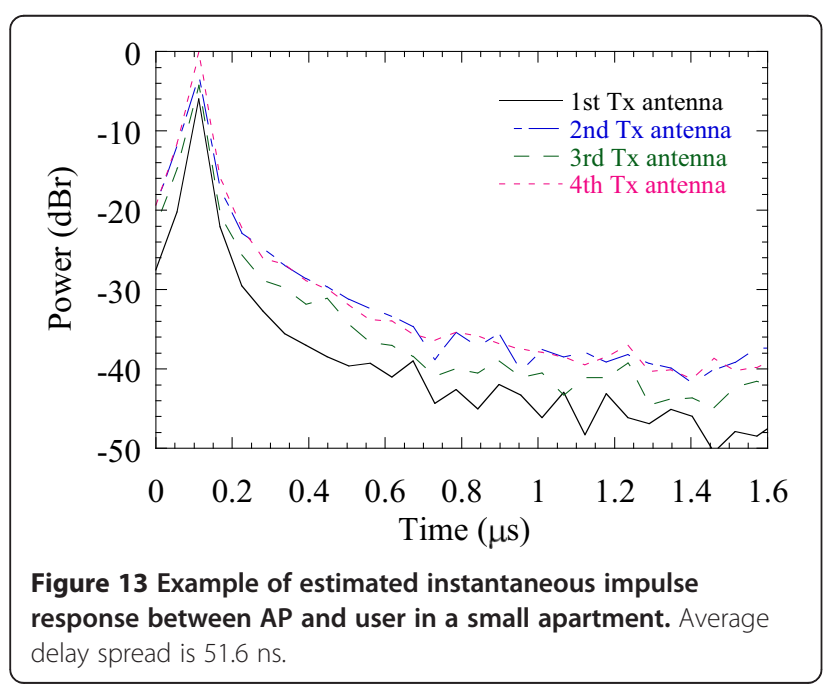

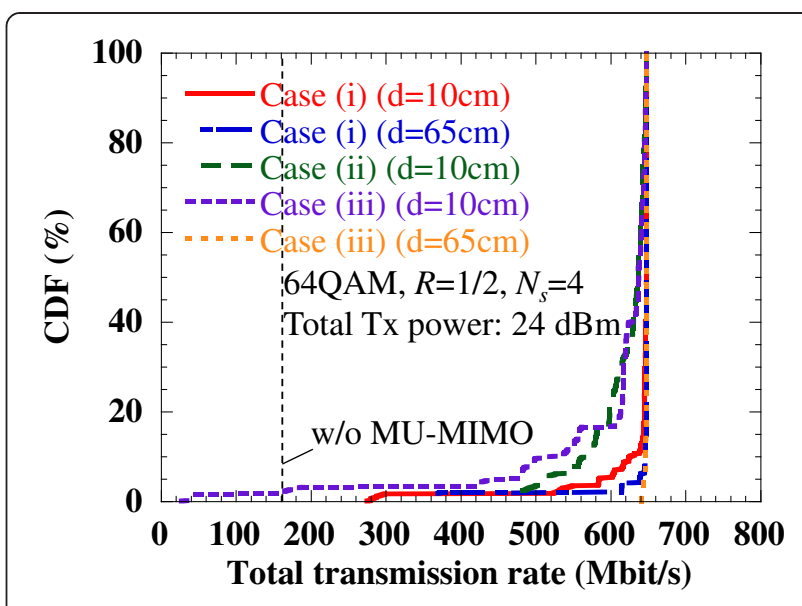

Figure 14 Effect of user locations. CDF of the transmission rate with the user location and distance as parameters when there are four users.

and the users (i), to minimize it (ii), or to square the user distribution (iii). Figure 13 shows an example of the estimated instantaneous impulse response in an apartment environment. As the figure shows, the measured value of the delay spread in the apartment is $51.6 \mathrm{~ns}$, smaller than that in the office and experimental room environments. This is because the apartment is of wooden construction, and the direct wave through a wall is dominant. Figure 14 plots the cumulative distribution function (CDF) of the transmission rate with the user location and distance as a parameter when there are four users. The modulation scheme and coding rate were set to 64QAM and $R=1 / 2$, respectively. When the distance between the adjacent users $(d)$ was $10 \mathrm{~cm}$, the transmission rate in the user location scenario (i) reaches the theoretical maximum transmission rate $(648 \mathrm{Mbit} / \mathrm{s})$ at a $10 \% \mathrm{CDF}$ for this transmission parameter. In contrast, the transmission rates in scenarios (ii) and (iii) are slightly lower. This is because

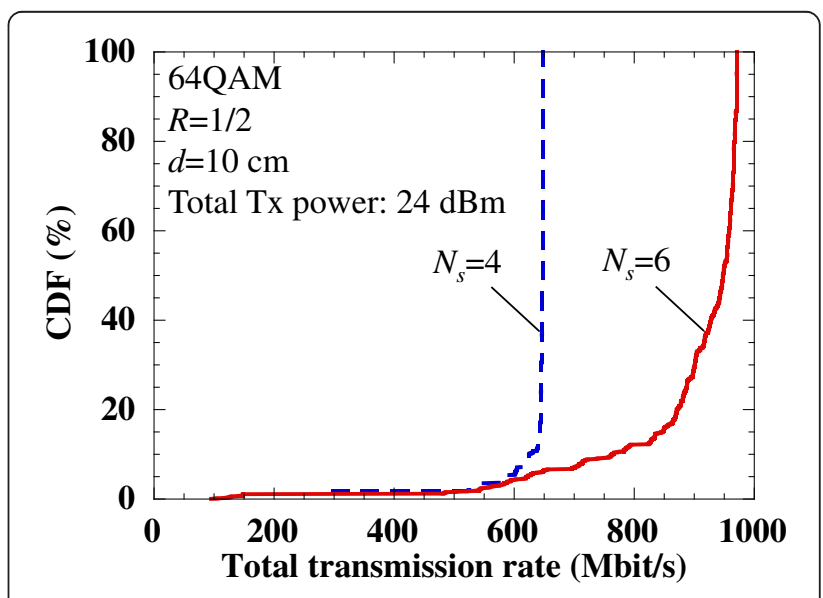

Figure 15 Effect of the number of users. CDF of the transmission rate when there are four or six users in scenario (i) when $d$ was $10 \mathrm{~cm}$. 
the channel correlation between the users becomes larger than that in scenario (i), and the orthogonality of the transmitted signals between users is deteriorated. However, 520 and $560 \mathrm{Mbit} / \mathrm{s}$ transmission rates can be respectively obtained at a $10 \% \mathrm{CDF}$ in scenarios (ii) and (iii), and they are much larger than those of the TDMA downlink transmission without MU-MIMO. Furthermore, when $d$ reaches $64 \mathrm{~cm}$, the transmission rate in both scenarios (i) and (iii) reaches the theoretical maximum transmission rate, although scenario (iii) is a close-packed structure. Figure 15 plots the CDF of the transmission rate when there are four or six users in scenario (i) when $d$ was $10 \mathrm{~cm}$. As the figure shows, throughout the whole range, the transmission rate is higher for six users than for four, and $948 \mathrm{Mbit} / \mathrm{s}$ transmission rates can be achieved with six users at a $50 \%$ CDF. Therefore, the DL MU-MIMO is also valid in such an environment with a small delay spread. The total transmission rate with four users reaches the theoretical maximum transmission rate at a $10 \% \mathrm{CDF}$. In contrast, the total transmission rate with six users reaches it at a 95\% CDF. However, the total (per user) transmission rates of 640 (160) and 780 (130) Mbit/s with four and six users can be achieved at a $10 \% \mathrm{CDF}$, and it is found that the number of multiplexed users increases and the total transmission rate improves.

\section{Conclusion}

This paper presents the indoor experimental results obtained for real-time DL MU-MIMO-OFDM transmission using a RIMC scheme in gigabit wireless LAN systems. In the proposed scheme, the inverse of the autocorrelation matrix can be calculated in the arrival order of CSI feedback frames from each user in a TDMA manner, resulting in reduced overhead for the transmit beamforming weight computation. In this paper, we evaluated the effects of the delay spread of the propagation channels and the time variations in three places with different channel characteristics and verified the feasibility of DL MU-MIMO-OFDM for gigabit-per-second transmission in wireless LAN systems. The experiment results showed that real-time DL MU-MIMO transmission of $972 \mathrm{Mbit} / \mathrm{s}$ was achieved with zero forcing-based transmit beamforming obtained by the proposed RIMC scheme at the AP and a simple decoder with each single-antenna user. In measuring the transmission rate in actual indoor environments, we found that a total transmission rate of $780 \mathrm{Mbit} / \mathrm{s}$ was achieved with six users at a $10 \% \mathrm{CDF}$. The obtained results confirm that DL MU-MIMO-OFDM is a promising technology to enable gigabit-per-second transmission for simple terminals with feasible hardware implementation at the AP.
Received: 11 December 2012 Accepted: 11 June 2013

Published: 27 June 2013

\section{References}

1. E Perahia, IEEE 802.11 n development: history, process, and technology. IEEE Commun. Mag. 46(7), 48-55 (2008)

2. A Goldsmith, SA Jafar, N Jindal, S Vishwanath, Capacity limits of MIMO channels. IEEE JSAC 21(5), 684-702 (2003)

3. QH Spencer, AL Swindlehurst, M Haardt, Zero-forcing methods for downlink spatial multiplexing in multiuser MIMO channels. IEEE Trans. Signal Processing 52, 461-471 (2004)

4. LU Choi, RD Murch, A transmit preprocessing technique for multiuser MIMO systems using a decomposition approach. IEEE Trans. Wireless Commun. 3, 653-668 (2004)

5. Z Shen, R Chen, JG Andrews, RW Heath Jr, BL Evans, Low complexity user selection algorithms for multiuser MIMO systems with block diagonalization. IEEE Trans. Signal Processing 54, 9 (2006)

6. 802.11 Working Group of the IEEE 802 Committee, P802.11ac/D5.0, Draft Standard for Information Technology-Telecommunications and Information Exchange Between Systems: Local and Metropolitan Area Networks_-Specific Requirements Part 11: Wireless LAN Medium Access Control (MAC) and Physical Layer (PHY) Specifications: Enhancements for Very High Throughput for Operation in Bands below $6 \mathrm{GHz}$ (IEEE, New York, 2013)

7. RV Nee, Breaking the gigabit-per-second barrier with 802.11ac. IEEE Wireless Commun. 18(2), 4 (2011)

8. T Katser, A Wtlzeck, M Berentsen, M Rupp, Prototyping for MIMO systems-an overview (Paper presented at the 12th EUSIPCO, Vienna, Austria, 2004)

9. O Font-Bach, N Bartzoudis, A Pascual-Iserte, DL Bueno, A real-time MIMO-OFDM mobile WiMAX receiver: architecture design and FPGA implementation. Comp. Netw. 55(16), 3634-3647 (2011)

10. C Caban, C Mehlfuhrer, R Langwieser, AL Scholtz, M Rupp, Vienna MIMO testbed. EURASIP J. Appl. Signal Proces. 2006, 1-13 (2006)

11. F Ludwig, A Budweg, S Paul, FPGA implementation of ZF-THP for MU-MISO-OFDM systems (Paper presented at the 17th international OFDM workshop 2012 (InOWo '12), Essen, Germany, 2012)

12. RA Monzingo, TWM Miller, Introduction to Adaptive Arrays (Wiley, New York, 1980)

13. K Ishihara, Y Asai, R Kudo, T Ichikawa, M Mizoguchi, Indoor experiments on real-time multiuser MIMO transmission in wireless LAN systems (Paper presented at IEEE Wireless Communications and Networking (WCNC), Paris, France, 2012)

14. Xilinx Inc, The Xilinx Inc. homepage, 2013. http://www.xilinx.com/. Accessed 18 March 2013

15. IEEE Computer Society, IEEE Standard for Information TechnologyTelecommunications and Information Exchange Between Systems: Local and Metropolitan Area Networks-Specific Requirements Part 11: Wireless LAN Medium Access Control (MAC) and Physical Layer (PHY) Specifications: Enhancements for Higher Throughput (IEEE, New York, 2009)

16. JG Proakis, Digital Communications, 4th edn. (McGraw-Hill, New York, 2001)

17. P Robenson, S Kaiser, Analysis of the effects of phase-noise in orthogonal frequency division multiplex (OFDM) systems (Paper presented at the IEEE international conference on communications (ICC), Seattle, USA, 1995)

18. M Joham, W Utschick, JA Nossek, Linear transmit processing in MIMO communications systems. IEEE Trans. Signal Proces. 53(8), 2700-2712 (2005)

19. H Lutkepohl, Handbook of Matrices (Wiley, New York, 1996)

doi:10.1186/1687-6180-2013-123

Cite this article as: Ishihara et al.: Development and experimental validation of downlink multiuser MIMO-OFDM in gigabit wireless LAN systems. EURASIP Journal on Advances in Signal Processing 2013 2013:123. 